Cancio Ferruz, Arturo.

Personal Investigador en Formación, Departamento de Dibujo, Facultad de Bellas Artes, Universidad del País Vasco - UPV/EHU.

\title{
El arte ya no existe, o no existe todavía...el arte falta.
}

TIPO DE TRABAJO

Comunicación virtual.

PALABRAS CLAVE

Arte, cultura de consumo, economía, competitividad, comunicación publicitaria.

KEY WORDS

Art, consumer culture, economics, competitiveness, advertisement, communication.

\section{RESUMEN}

El título de este texto parafrasea una idea de Deleuze ${ }^{1}$. En efecto, el arte aparece como una ausencia de la cultura presente en la que nos vemos sumergid*s. Proliferan convocatorias que requieren la presencia del arte, aunque bajo condiciones que mantienen la práctica artística dentro de lo posible, pero bastante lejos de lo real. El arte precisa de una dimensionalidad imposible de concebir por los "regios despliegues del imperio (que) garantizan que la contingencia se convierta en necesidad y no derive en desorden" ${ }^{2}$. Tanto instituciones públicas, como privadas presentan sus programas de creatividad artística, que implican el sometimiento a ciertos valores, que se ponen en funcionamiento mediante tres mecanismos de control principalmente; la concurrencia competitiva, el juicio monetario y el impuesto de comunicación.

Este texto expone estos tres mecanismos de control, que mantienen a la creatividad artística en una estado de virtualidad sostenida; "la realidad de lo virtual, con unos eficaces efectos generados por algo que no existe todavía en su plenitud" ${ }^{3}$. Se presentan además algunos trabajos de creación, como muestra de "virtualidades singulares que operan la conexión entre lo posible y lo real" ${ }^{4}$ que, sin replegarse a los espacios canónigos del Arte, lo hacen en una posición de resistencia, transformando los lugares que ocupan y deconstruyendo estos mecanismos de control sistémicos, subvirtiendo además sus valores.

\section{ABSTRACT}

The title of this paper paraphrases an idea from Deleuze ${ }^{5}$. In effect, art appears as an absence from the present culture we are sinked in. Different calls proliferate, requiring art to be present under conditions that make artistic practice be within the realm of possibility, but really far from being actual. Art needs a dimensionality impossible to be conceived by the "royal deployments of Empire (that) guarantee the contingency becomes a necessity and does not descend into disorder" . Public and private institutions present their programmes of artistic creativity, that imply being subjected to some values, that are set up mainly through three mechanisms os control; competitive tender, monetary judgement and communication tax.

\footnotetext{
${ }^{1}$ La frase original dice así: "...el pueblo ya no existe, o no existe todavía... el pueblo falta" en Deleuze, G.: La imagen-tiempo. Estudios sobre cine 2. Ed. Paidós, Barcelona, 1987, 287.

${ }^{2}$ Hardt, M. y Negri, A.: Imperio. Ed. Paidós, Barcelona, 2002, 325.

${ }^{3}$ Žižek, S.: The reality of the virtual, 2004 [https://www.youtube.com/watch?v=RnTQhIRcrno]. [8/04/2015].

${ }^{4}$ Hardt, M. y Negri, A.: Imperio. Ed. Paidós, Barcelona, 2002, 336.

${ }^{5}$ The original sentence states: “...el pueblo ya no existe, o no existe todavía... el pueblo falta” en Deleuze, G.: La imagen-tiempo. Estudios sobre cine 2. Ed. Paidós, Barcelona, 1987, 287.

${ }^{6}$ Hardt, M. y Negri, A.: Imperio. Ed. Paidós, Barcelona, 2002, 325.
} 
This paper exposes these mechanisms of control, that keep artistic creativity in a state of contained virtuality; "the virtuality of the real, with efective effets generated by something that doesn't fully exist" ${ }^{\prime 7}$. Some works of creation are also presented as samples of "singular virtualities that operate the connection betwen the possible and the real" and, without become withdrawn into the traditional spaces of Art, transform the places they occupy, deconstruct these mechanisms of control, and subvert their values.

\section{CONTENIDO}

\section{INTRODUCCIÓN}

El presente texto está basado en una labor previa de recopilación y estudio de alrededor de cincuenta iniciativas, a través de las cuales artistas son convocados para exhibir su trabajo fuera de los canales convencionales del arte: colecciones, museos, galerías, salas de exposiciones,... Las convocatorias seleccionadas se caracterizan por su interés en promover actividades artísticas destinadas a su difusión en establecimientos comerciales, como tiendas, grandes almacenes, centros comerciales,... Se presta especial atención en este estudio a las condiciones de participación y las bases reguladoras presentada por sus organizador*s: instituciones y empresas públicas y privadas fundamentalmente.

El alcance de este estudio, aún en desarrollo, es internacional, y atiende a aquellas convocatorias que promueven la creación de "sinergias entre la practica a artística y la actividad comercial" ${ }^{8}$, en el periodo comprendido entre los años 2005 y 2015 , definiendose de este modo el contexto objetivo. Al no disponer todavía de datos concluyentes, en el presente trabajo no se presentan resultados objetivos, sino más bien focos de interés que apuntan a la existencia de tres mecanismos de control, en las condiciones de participación en estas convocatorias: la concurrencia competitiva, el juicio monetario y el impuesto de comunicación, que al ponerse en funcionamiento, tienen una incidencia en el valor y el carácter de las prácticas artísticas desarrolladas en este contexto.

Con el fin de ofrecer una anticipación del estudio, se ha llevado a cabo una selección de trabajos, realizados por artistas en establecimientos comerciales, en cuyo análisis nos apoyaremos para desarrollar las hipotesis de partida. Se ha tenido en cuenta a la hora de elegir esta metodología, una tesis reciente que sostiene que: algunos trabajos en arte, al desarrollarse vinculados a los lugares y la cultura del consumo de masas, se constituyen en "topoi que significan a la sociedad y las estructuras que significan", ya que "las estrategias del consumo -reproductora de un estado de las cosas - se relacionan con una cierta instanciación de la estética, la ética y la política y, consecuentemenmte, se produce una cierta instanciacion del objeto, el sujeto y la comunidad." ${ }^{\prime 9}$

Con esta motivación, la instalación interactiva Instant Art Carreer (2013), de Niklas Roy y Kati Hyyppä, es contemplada como un modelo condensado, que nos revela una visión de la mayoría de las caracteristicas de participación que son planteadas en las convocatorias objeto de estudio. Mientras que, la Free Sculpture (2010) de Diego Vivanco y Benjamin Bernard, y la acción de Pli Sotic, Carnefour (2008), demuestran que "lo virtual puede ejercer presión en los límites de lo posible y así alcanzar lo real. " ${ }^{10}$

\section{INSTANT ART CARRER}

Para sumergirnos en el contexto voy a presentar, en primer lugar, la instalación interactiva Instant Art Career ${ }^{11}$, que se presenta en la tercera edición del Street Art Festival ${ }^{12}$ de Katowice, Polonia, en 2013. A diferencia del resto de las intervenciones realizadas en este festival, que tienen lugar exclusivamente en la vía publica, Instant Art Carrer demuestra cierta ambiguedad al desarrollarse simultaneamente tanto en la calle, como en un espacio privado. Pero no solo esto resulta digno de atención, la organización del festival convoca, con la instalación de Roy e Hyypä, a toda la ciudadania y visitantes de la ciudad, a representar uno de los resúmenes posibles de lo que significa el Arte del siglo XX y su evolución, en un establecimiento comercial abandonado.

\footnotetext{
7 Žižek, S.: The reality of the virtual, 2004 [https://www.youtube.com/watch?v=RnTQhIRcrno]. [8/04/2015].

${ }^{8}$ Bases reguladoras Arteshop 2015, p.: 3. [http://www.arteshopbilbao.com/2015/es/pdf/bases-reguladoras.pdf]. [5/05/2015]

${ }^{9}$ Barros, C.: La obra de Arte frente al (super) mercado: ética, estética, política y consumo en el Cono Sur. Proquest, $2010,3,4$.

${ }^{10}$ Hardt, M. y Negri, A.: Imperio, Ed. Paidós, Barcelona, 2002, 326.

${ }^{11}$ Roy, N e Hyypä, K.: Instant art carrer, 2013 [https://youtu.be/fhL3XgkMiMk].[5/05/2015]

${ }^{12}$ Este festival cuenta con el apoyo del gobierno municipal de esta ciudad, así como de un numeroso grupo de patrocinadores privados. Sus organizadores invitan anualmente, desde 2011 y hasta este mismo año, a artistas punteros a nivel mundial en Street Art, para intervenir en el paisaje urbano de la ciudad. [http://katowicestreetartfestival.pl/]. [05/05/2015]
} 
Instant Art Carrer, a modo de palimpsesto, plantea una convocatoria dentro de la convocatoria del festival, e implementa un artefacto para finalmente desvelar, sintetizandolo, el proceso de mercantilización del Arte. Este artefacto funciona en tres fases, a traves de las cuales tanto el establecimiento comercial en el que se dearrolla el proceso, como I*s actores y actrices participantes, se van transformando tanto fisica, como conceptualmente. En primer lugar, la organización proporciona los medios de producción sofisticados, aunque limitados- de acceso publico, pero de propiadad privada. Aceptando estas condiciones, los ciudadanos-artistas se ponen manos a una obra situada fuera de su alcance, al otro lado del muro de cristal.

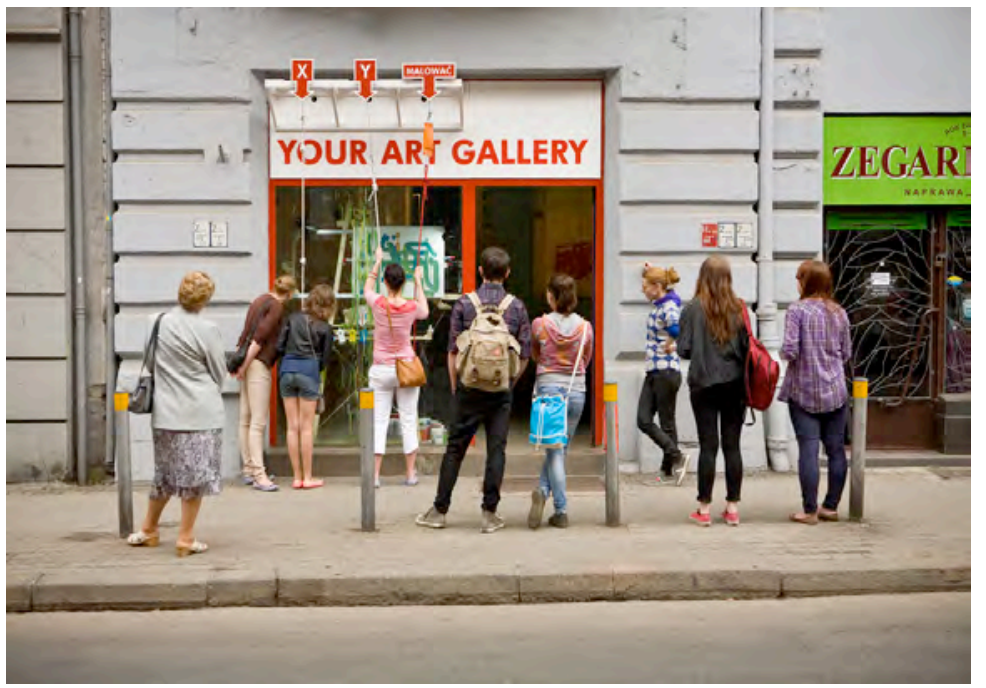

A pesar de los medios precarios, aunque tecnologicamente avanzados, que el sistema les proporciona, I*s artistas instantáne*s son capaces de realizar la fuerza de trabajo necesaria para que, una vez finalizado el proceso de producción, el taller pase a la segunda fase. Las pinturas realizadas por estos artistas, sin remuneración alguna, pasan a formar parte de una exposición dentro del mismo local abandonado-taller, ahora galería de arte instantáneo. Esta transformación implica una intervención curatorial, que no se hace pública, no se explicita, y que provoca que en estas pinturas realizadas colectivamente quede - también - oculto el trabajo de much*s de sus autor*s, debido a la superposición de capas de pintura sucesivas en los lienzos. La cara visible de esta carrera artística instantanea se obtiene valiendose de I*s propi*s ciudadan*s-artistas que, como autentic*s artistas del superstar system, son entrevistad*s con el fin de promover su producción en arte. No cabe duda de que en este procedimiento de producción de capital simbólico, social y cultural, existe un importante contraste, ya que es físico además de conceptual: lo que se hace arte aparece como velado, en privado y al otro lado del vidrio separador; mientras que el artista permanece en el espacio público. De hecho I*s artist*s son el propio público y, como veremos más delante, este hecho tiene mucha importancia a la hora de exponer uno de los mecanismos de control hacia los que estamos enfocando: la aplicación de un impuesto de comunicación en el proceso de creación artística.

En la tercera fase, el establecimiento comercial abandonado de Katowice, en su proceso de transformación, pasa a ser una sala de subastas de obras de arte instantáneo. Sus propietarios son los mismos que proporcionan los medios de producción, gestionan y administran la labor curatorial, los medios de comunicación, la sala de exposiciones y su archivo. En un proceso abierto a toda la ciudadania, incluso los artistas instantáneos pueden pujar por sus propias creaciones, convirtiendose en coleccionistas. El dinero recaudado por la venta de las pinturas es repartido entre I*s artistas creador*s de las obras. Se conforma así una suerte de bucle ontológico prodigioso, en la transformacion del capital simbólico en capital económico. En esta conformación entran en juego dos procesos contrastados:uno velado y otro visible, pero de características similares: ambos están relacionados con un mecanismo de concurrencia competitiva, y estrechamente vinculado al juicio económico que se plantean también como mecanismos de control objetivos de la investigación. Ambos serán amplíados más adelante.

\section{CONVOCATORIAS POSIBLES}

Instant Art Carrer se presenta como una convocatoria-modelo, que lleva asimiladas muchas de las condiciones de participación de la mayoría de las convocatorias estudiadas. Establecimientos comerciales e instituciones públicas y privadas ajenas a la esfera del Arte pujan por esos artes - es*s artistas - que ya hace décadas superaron los limites que les siguen imponiendo el tradicional universo de actrores y lugares, para intentar mantener su estatus elitista y exclusivo. Incontenible, el gran valor del Arte es solicitado por nuevos actores, hecha ya tácita esta noción en diferentes culturas. La producción, promoción y el consumo de arte debido a “...alguna clase de 
creencia común en su elevado valor"13, resultan tentadores. Esta creencia, o ley del deseo, manda, se hace cuerpo, y además tiene efecto contagio: muchas convocatorias aparecen en un punto-origen desde el cual se van extendiendo, salvo puntuales excepciones, en el tiempo y en el espacio.

También hay que tener en cuenta que el exceso de producción y consumo cuasi-cuantico propio del sistema capitalista imperante, estimula la abundancia de prácticas artísticas enfocadas a una sociedad ávida de imágenes repetidas y diferentes. A lo largo de la Historia del Arte, abundan los ejemplos de artistas que se han aprovechado del imaginario o los lugares de la cultura de consumo, para realizar sus trabajos. Solo algunos de estos los elegidos a ser destinados a los canales convencionales del Arte, ya que su metodología de archivo resulta inhabil a la hora de asumir la fluidez reproductiva actual. Es razonable que exista un numeroso grupo de artistas que, debido a “...su propia vanidad, estar hipnotizados por alguna clase de imperativo de autoría super-estrella que promete reconocimiento a gran escala, o a las expectativas de la cultura..." ${ }^{14}$, alimenten estos programas, encontrando su significación en convocatorias como las que se ponen en cuestión en este texto. Pero, ¿̇en qué condiciones?.

En lo que concierne a su archivo, a diferencia del Arte, "la cultura de masas no tiene un archivo a su disposición"15, lo que confirma este proceso de ocultamiento o invisibilización de la mayoría de la obra de I*s artistas, que participan en estas convocatorias., tal y como se pone de manifiesto en Instant Art Carrer. Aunque realmente, esto no es un inconveniente, si atendemos a las voces que reclaman la necesidad de un "arte contemporaneo anónimo"16. Sin embargo, no es del todo así: en numerosas convocatorias, como en el modelo propuesto, un grupo de expertos invisibiliza la mayoría de las obras participantes, mientras destaca unas pocas mediante procesos curatoriales opacos o inexistentes. Este procedimiento, en el que I*s participantes compiten por una posición, no solo eclipsa las obras y artistas no seleccionad*s, sino que llega incluso hasta a eclipsar cualquier intento de que "... la propia materialidad de las obras de arte organice el esquema relacional ...."17 de la convocatoria, en su abundancia y diversidad. Las convocatorias basadas en la concurrencia competitiva consiguen de este modo significarse a si mismas, a las entidades y personas encargadas de su organización y a un reducido y selecto numero de artistas, en una logica del meritismo. Algo ya parcticado en el campo del Arte, con la diferencia de que el Arte se deleita ademas con el saboer que genera la actividad artistica.

El significarse a si mismas, sin significar el arte que contienen, no solo se consigue mediante este proceso competitivo, existen además otros factores a tener en cuenta. En primer lugar, la falta de regulación laboral y de los derechos de I*s artistas implicados en la mayoría de estas convocatorias, y los presupuestos, mínimos o nulos, que presentan sus programas de actuación, para compensar el trabajo realizado por I*s artistas. Estos dos factores son determinantes a la hora de sostener que estas convocatorias consiguen la simbolización de un colectivo a costa de la precarización de una parte de el. Y se da en esa transformación de lo simbólico, a lo monetario, en ese bucle ontológico prodigioso que da el capital y que queda desvelado en Instant Art Carrer. Por otro lado, este juicio monetario - simbólico - puede, si acaso, resultar todavia menos ecuánime. Hay que tener en cuenta que son I*s propi*s participantes, artistas y comerciantes, I*s que junto con sus allegados y el público visitante e invitado, proporcionan el material visual, relacional - que es recaudado como impuesto de comunicación, y que mantiene viva la difusión y reproducción del programa , alimentando a los medios de comunicación empleados, en clara competencia, de nuevo, con I*s artistas.

Me parece importante concluir esta sección señalando, que la instalación Instant Art Carrer muy bien podría considerarse una forma de arte contemporáneo, como crítica al sistema del Arte, desde fuera de este sistema. Sin embargo, al consistir en una representación, permanece en un estado de ambigüedad, que la hace sospechosa de ser un intento de reproducción de un sistema preexistente, aunque en otro contexto. No obstante, esta descontextualizción es manifiestamente satatisfactoria subvirtiendo su sentido, ya que permite desvelar algunas de las condiciones reales del sistema del Arte. Al representase como una herramienta crítica que no presenta, ni parece prever, herramientas para la elaboración de otras críticas posibles, cualquier discrepancia emergente puede fácilmente ser una vez más - velada, e incluso hasta reabsorbida en una comisión de connivencia. Afortunadamente, Instant Art Carrer resultó un éxito de ventas y consiguió recaudar ciertos fondos, que revirtieron en I*s artistas praticipantes, en un reparto de capital monetario, que los propios Roy y Hyyppä señalan como un arte en si mismo. No dudamos que seguramente lo fue.

\section{VIRTUALIDADES SINGULARES}

Es tiempo ya de aceptar la proposición de que el arte precisa de una dimensionalidad imposible de concebir por los "regios despliegues del imperio (que) garantizan que la contingencia se convierta en necesidad y no derive en desorden"18. Se han desvelado los tres mecanismos de control, que mantienen a la creatividad artística en una estado de virtualidad sostenida y ahora, por un lado, surgen

\footnotetext{
${ }^{13}$ Aranda, J. et al.: Are you working too much?: Post-fordism, precarity, and the labor of art. Sternberg, 2011, 6.

${ }^{14}$ Aranda, J. et al.: Are you working too much?: Post-fordism, precarity, and the labor of art. Sternberg, 2011, 6.

${ }^{15}$ Groys, B.: "The artist as consumer". En Shopping. A century of Art and Consumer Culture. Hatje Cantz, 2002, 59.

${ }^{16}$ Badiou, A.: Las condiciones del arte contemporaneo, 2013. [https://youtu.be/0Jpqoice0rc].[05/05/2015]

${ }^{17}$ Vergara, L.: "El esquema de una organización". En 24ª exposición visual-sonora. Facultad de Bellas Artes - UPV/EHU, $2012,9$.

${ }^{18}$ Hardt, M. y Negri, A.: Imperio. Ed. Paidós, Barcelona, 2002, 325.
} 
voces que entienden que "esta idea de un "alto valor" que preside, y alimenta de verdad, una idea de trabajo en arte como trabajo gratis, se deben contestar" ${ }^{19}$ Es el juicio economico el que hay que poner a cero, y en equilibrio estructural, como sugiere una de las free sculptures realizadas con carros de la compra de hipermercados.

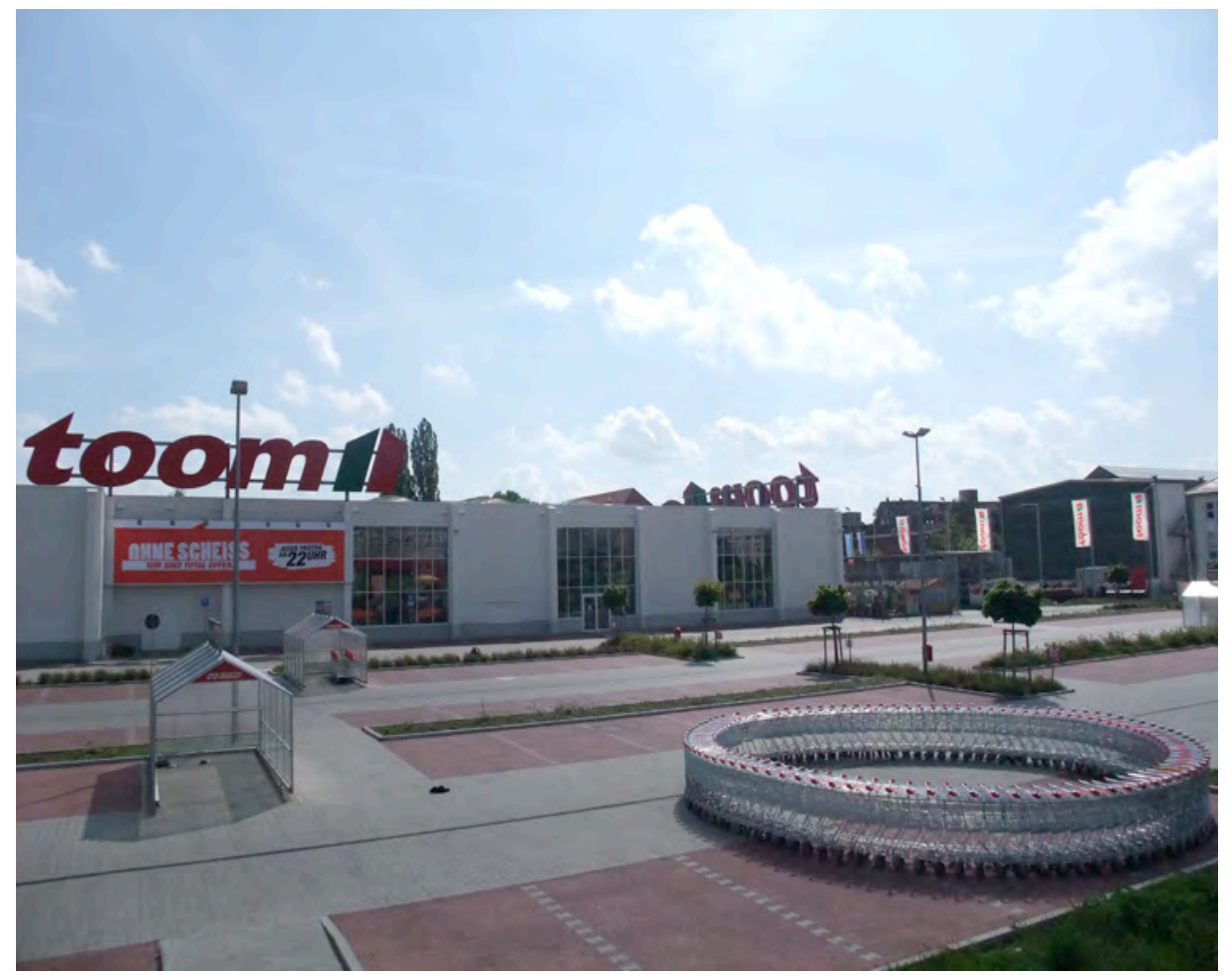

Los artistas Diego Vivanco y Benjamín Bernard realizaron esta escultura aun inédita, y que forma parte de una serie , como una práctica artística proactiva, autogestionada y sin patrocinio alguno. La pieza nos habla del bucle ontológico prodigioso y, para poder realizarla, los artistas invirtieron la misma moneda que recuperaron, una vez que la escultura estuvo acabada. Pero en su título, Free Sculpture, no solo se refirere a que es una escultura gratis. Aunque es su gratuidad, precisamente, la que hace rebosar de sentido su otro significado: es una escultura libre. La forma de la escultura y su imagen, junto con su proceso de producción, nos presentan una estructura rotunda que da cuenta de lo poco que se necesita, para lo mucho que se puede y lo nada que se aprovecha. Su escultura se presenta como lo que para la cultura de consumo representa el arte, gracias al lugar empleado por los artistas para llevarla de lo virtual, a lo posible y aquí a lo real, para luego registrarla in situ. Un cero es representación de la no-dimension. El arte cae aquí, al ser considerado adimensional , como una más de las mercancias que circulan y se asimilan a la dimensión simbólica del sistema económico monetario. No obstante se levanta, si tenemos en cuenta que la pieza es anónima, finita, repetible, y además evidencia no solo una promesa de lo posible, sino una realidad hecha posible desde una virtualidad singular, a traves de lo ecónomico monetario, que resulta en algo fútil. Esta revelación de las competencias y la promocion de las economias de lo monetario y de lo no, y las relaciones entre ellas, es ya urgente tener en cuenta de un modo crítico, si acaso en la práctica artista en este contexto de la cultura comercialque nos ocupa.

De competencia y promoción nos habla la acción de la artista Pli Sotic titulada Carnefour. El registro de vídeo, aun sin publicar, nos presenta una acción de 2008 en la que se puede ver a la artista entrando en un supermercado con un carro de la compra, para vaciar una de las estanterías de comida para perros y colocarse ella misma dentro. La artista con su acción nos presenta, una "movilización particular que ejerce la obra de arte sobre una cierta indistinción entre la estética del mercado y la estética del arte" ${ }^{20}$. Lo mediatico y lo artistico están en clara pugna como instrumentos para la difusión de los programas artísticos, en los lugares de la cultura de consumo que nos ocupan. Sotic denuncia que lo mediático, en muchas ocasiones, fagocita el saber y las imágenes del arte, para la realización de sus productos atendiendo a la lógica del mercado. Teniendo en cuenta lo del hombre es un lobo para el hombre, y sabiéndose con la batalla perdida, la artista se somete y se ofrece segun sus propias palabras como carne de artista para publicista.

\footnotetext{
${ }^{19}$ Aranda, J. et al.: Are you working too much?: Post-fordism, precarity, and the labor of art. Sternberg, 2011, 6.

${ }^{20}$ Barros, C.: "La broma de Raul: Objeto, mercancía y estética en Tajos de Rafael Courtoise”. En Revista de Crítica Literaria Latinoamericana, 1er semestre de 2010, Año XXXVI, n. ${ }^{\circ}$ LXXI, 315
} 


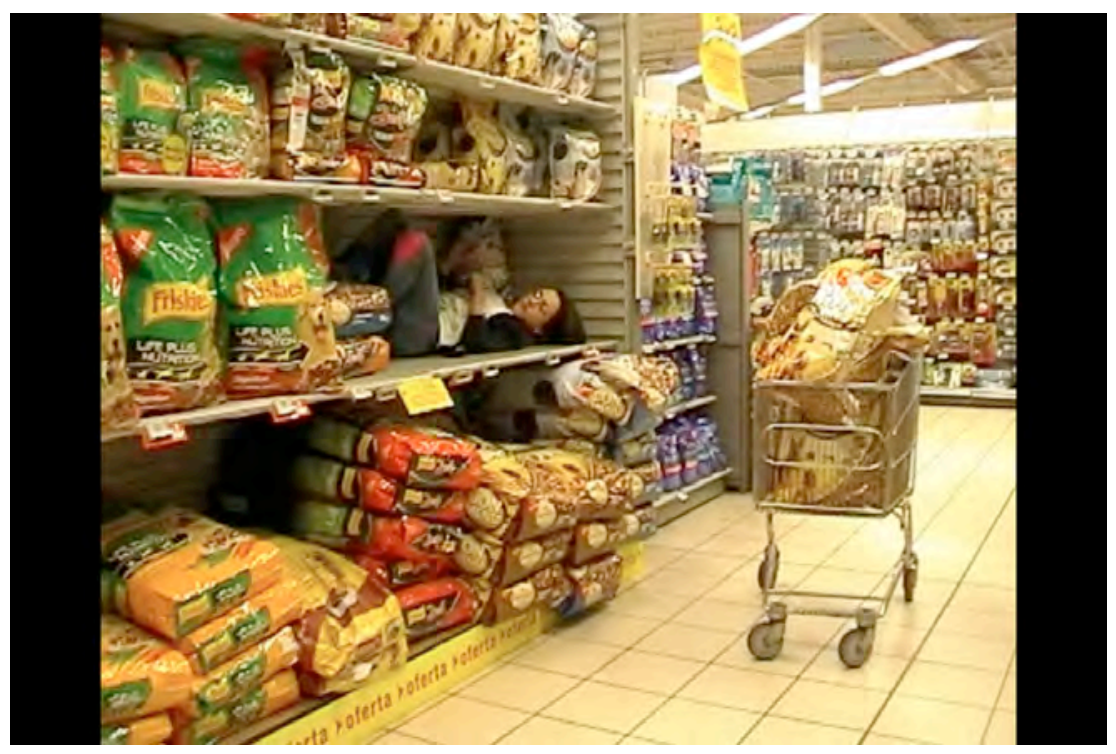

Operar en una posición no ya de resistencia, sino de guerrilla, implica el compromiso de transformar los lugares que se ocupan, deconstruir los mecanismos de control sistémicos que se detecten y subvertir sus valores. Resulta difícil de concebir que posiciones como esta que nos presenta Sotic, se asimilen en programas de creatividad artística enfocados hacia "la realidad de lo virtual, con unos eficaces efectos generados por algo que no existe todavía en su plenitud" ${ }^{21}$. Y si fuera concebible y posible, resultaría mucho más dificil evitar que una acción como Carnefour pase de ser una presentacion, a una representacion, al efectuar tal cambio de contexto. Asimismo, las convocatorias analizadas no parecen ofrecer las condiciones que numeros*s artistas precisan para en su proceso de encuentro con esa "virtualidad singular, que opera la conexión entre lo posible y lo real" ${ }^{22}$. ¿Nos encontramos realmente en callejones sin salida? 0 ¿Es posible que se trate de una encrucijada más del campo expandido del arte?

\section{CONCLUSIONES}

El trabajo de Niklas Roy y Kati Hyyppä evidencia la existencia de tres mecanismos de control en las condiciones de participación en estas convocatorias estudiadas: la concurrencia competitiva, el juicio monetario y el impuesto de comunicación. Tambien hemos podido comprobar como estos mecanismos de control tienen una incidencia en el valor y el carácter de las prácticas artísticas desarrolladas en este contexto. Es este un valor virtual mediante el cual se enmascaran y aplanan la multidimensionalidad de las propuestas artística. Las relaciones entre la produccion y el consumo de arte en este contexto, ven reducidos sus valores simbolicos reales a aquellos permitidos por imposición mediatica .

No obstante, el balance simbolico entre el objeto artístico y su valor monetariose ve deasafiado por numerosas tomas de posicion por parte de artistas, que optan por poner en evidencia la realidad del intercambio simbólico que proporciona el objeto artístico, y que es sustituida por el valor simbólico del dinero gracias a su supuesta eficacia. En las convocatorias que hemos entrado a debatir, lo imaginario, lo simbolico y lo real permanecen en una realidad todavia virtual que no es efectiva para el arte.

\footnotetext{
${ }^{21}$ Žižek , S.: The reality of the virtual, 2004 [https://www.youtube.com/watch?v=RnTQhIRcrno]. [8/04/2015].

${ }^{22}$ Hardt, M. y Negri, A.: Imperio, Ed. Paidós, Barcelona, 2002, 336.
} 


\section{FUENTES REFERENCIALES}

Aranda, J. et al.: Are you working too much?: Post-fordism, precarity, and the labor of art. Sternberg, 2011.

Badiou, A.: Las condiciones del arte contemporaneo, 2013. [https://youtu.be/OJpqoice0rc].[05/05/2015]

Barros, C.: La obra de Arte frente al (super) mercado: ética, estética, política y consumo en el Cono Sur. Proquest, 2010.

Barros, C.: "La broma de Raul: Objeto, mercancía y estética en Tajos de Rafael Courtoise”. En Revista de Crítica Literaria Latinoamericana, 1er semestre de 2010, Año XXXVI, n.o LXXI.

Bases reguladoras Arteshop 2015. [http://www.arteshopbilbao.com/2015/es/pdf/bases-reguladoras.pdf]. [5/05/2015]

Deleuze, G.: La imagen-tiempo. Estudios sobre cine 2. Ed. Paidós, Barcelona, 1987.

Groys, B.: "The artist as consumer". En Shopping. A century of Art and Consumer Culture, Hatje Cantz, 2002.

Hardt, M. y Negri, A.: Imperio, Ed. Paidós, Barcelona, 2002.

Roy, N e Hyypä, K.: Instant art carrer, 2013. [https://youtu.be/fhL3XgkMiMk].[5/05/2015]

Vergara, L.: "El esquema de una organización”. En 24̣ exposición visual-sonora. Facultad de Bellas Artes - UPV/EHU, 2012.

Žižek, S.: The reality of the virtual, 2004. [https://www.youtube.com/watch?v=RnTQhIRcrno]. [8/04/2015]. 